\title{
Estimating the global power consumption in communication networks
}

\author{
Sofie Lambert, Ward Van Heddeghem, Willem Vereecken, Bart Lannoo, Didier Colle, Mario Pickavet \\ Department of Information Technology, Ghent University - IBBT, Gent, Belgium, \\ sofie.lambert@intec.ugent.be
}

\begin{abstract}
This presentation will estimate the worldwide power consumption and carbon footprint of communication networks and compare this with other ICT fields such as data centres and personal computers. This paper concentrates on explaining the used methodology.
\end{abstract}

\section{Introduction}

In recent years the power efficiency of communication networks has received a lot of research attention. Various strategies have been proposed to reduce the power consumption of both mobile and fixed networks on all levels, from the access network to the core ${ }^{1,2,3}$.

In most studies, potential energy savings are expressed as a percentage of the power consumption of current technologies, or power consumption values per user for certain access rates are provided. To assess the global impact of these savings in absolute numbers, an estimate is required for how much power present-day networks consume as a whole.

Often cited values for the power consumption of communication networks date from five to ten years ago or are extrapolations based on these valuesi. Fiber rollout, smart devices requiring mobile internet access and rapid customer base growth in emerging markets are likely to have transformed the power consumption in networks in these last few years. Our aim is to calculate an updated value for the network share of global energy consumption in 2012.

\section{Bottom-up versus top-down approach}

Many studies on the electricity consumption in communication networks use a bottom-up approach, where the electricity consumption of individual components of the network is added up to get an idea of the total consumption.

Kilper et al. ${ }^{6}$, for example, use a transactionbased approach. The power per bit rate is calculated by adding up the power of all the equipment in the network that is used to deliver

\footnotetext{
${ }^{\mathrm{i}}$ Our previous report on the worldwide energy needs for ICT ${ }^{4}$ was based on data from 2007. In the Smart2020 report ${ }^{5}$, the network section of the analysis used reported energy consumption values of telecom providers in 2002, which were extrapolated based on the expected increase in accounts in 2002-2020.
}

a given service on a mean transaction basis. The access equipment considered is that of a Passive Optical Network (PON). This provides state-of-the-art estimates, which are useful for future projections, but may be too optimistic for present-day networks.

Baliga et al. ${ }^{7}$ also use a bottom-up approach: they estimate the electricity consumption per user for different access technologies by adding the energy consumption of three components (customer premises equipment, remote node or base station and terminal unit). The values for these components are based on manufacturers' data sheets.

Though the bottom-up approach is very useful when comparing different technologies, it is less suitable to get an idea of the global energy consumption in the network, since it is nearly impossible to consider every technology in use, every user profile, every geography, etc.

The approach we propose is top-down: we start from the total energy consumption of telecom providers and try to attribute this power consumption to different services. Based on these numbers, we estimate the global electricity consumption in communication networks.

\section{Calculation method for the operator side}

As a starting point, we list the top ten biggest telecom operators by mobile subscriptions and by (fixed) broadband subscriptions. The latter typically also have large market shares in fixed telephony and (IP)TV. For each of these operators, we try to gather the following information:

- total annual electricity consumption and breakdown of this consumption by activity (mobile network, fixed network, data centers, offices...)

- number of fixed telephone accesses 
Tab. 1: Worldwide subscriptions (in millions). Pay TV includes cable, satellite, terrestrial and IPTV.

2012 numbers are our own estimates.

\begin{tabular}{|c|c|c|c|c|c|c|}
\hline & 2007 & 2008 & 2009 & 2010 & 2011 & 2012 \\
\hline Fixed telephone access $^{8,9}$ & 1,255 & 1,250 & 1,249 & 1,228 & 1,205 & 1,182 \\
\hline Wired broadband ${ }^{8,9}$ & 346 & 409 & 465 & 528 & 590 & 650 \\
\hline Pay TV ${ }^{10}$ & 559 & 612 & 663 & 730 & 791 & 846 \\
\hline Mobile access ${ }^{8,9}$ & 3,372 & 4,034 & 4,650 & 5,315 & 5,975 & 6,615 \\
\hline
\end{tabular}

- number of fixed broadband accesses ${ }^{\mathrm{ii}}$

- number of (IP-)TV clients

- number of mobile accesses (this includes voice and data)

Based on numbers from different operators we can create a mathematical model where the power consumption for each operator $P_{\text {operator }}$ (excluding data centers and offices) equals

$\mathrm{P}_{\text {operator }}=\sum_{\text {service }}$ subscribers service $\times \mathrm{P}_{\text {avg,service }}$

where the services are fixed telephone, fixed broadband, TV and mobile access. By fitting this power consumption per operator to the reported values, we can determine $P_{\text {avg,service, the average }}$ power consumption per subscriber for each service

By multiplying these averages with the global numbers of subscriptions (Table 1) and adding everything up, we obtain an estimate for the global electricity consumption in communication networks ${ }^{\text {iii. }}$.

In our calculations, we leave out the electricity consumption for data centers, since our calculation of the power consumption in networks will be integrated in a study of the electricity consumption of ICT in general, in which data centers are considered separately.

\section{Calculation method for the user side}

In order to use the network, users need to have access equipment installed at home. In our network study, we will consider the energy consumption of set-top boxes and modems, since these are generally provided by telecom operators. The consumption of these devices is not included in the electricity bill of telecom providers, so we need to look at them separately. By combining average energy consumption values for modems and set-top boxes from other studies with our global

\footnotetext{
ii We assume the power consumption in the network due to narrowband connections is negligible compared to broadband.

iii The number of TV subscribers given in Table 1 is an overestimation, since it also includes clients of non-telecom operators. A more detailed breakdown is still to be determined.
}

subscriber numbers, we can estimate the global power consumption of these devices. Modems and set-top boxes have a relatively high power consumption 7,11 , so we expect them to have a significant impact.

\section{Challenges}

There are still several challenges to be tackled before we can obtain our final results, most of which are related to the (un)availability of data.

Though many telecom operators disclose their electricity consumption, it is hard to find data from operators in emerging markets such as India and China. Since approximately 75 percent of mobile customers are in these markets $^{12}$, not having data for these countries can make our results less accurate. Additionally, a study based on publicly available power consumption values may lead to overly optimistic results, since the companies that publish these values have typically made efforts towards more energy efficiency.

Another problem comes from the fact that incumbent operators often lease parts of their networks to other operators. This means that the number of customers connected to a network is not necessarily the same as the number of subscribers reported by the operator. We need to base our results on aggregated numbers rather than individual operators' electricity consumption if we want to cancel out this effect. Unfortunately, this means we can no longer use our mathematical model to obtain the average power consumption per subscriber for each service, since this relies on the fact that the power consumption per operator is proportional to the number of reported subscribers.

Some operators provide a breakdown of their power consumption for the mobile and fixed network, which allows us to estimate the relative importance of these two categories of services. Splitting up the power consumption among fixed services is a more difficult task, since they are transported over the same physical medium.

When looking at the aggregated numbers, we need to find a new strategy to extrapolate the power consumption to a global number. If we choose our sample so that the relative 
subscriber numbers for different services match the worldwide ratios, we can still extrapolate the data to obtain the global power consumption. For example, in 2010 there were 2.33 fixed telephone subscribers and 10.07 mobile subscribers for each broadband subscriber in the world. If we make sure our sample satisfies these relationships (and is sufficiently large), we can obtain an estimate for the global power consumption by multiplying the total power consumption of our sample with one factor, namely the ratio of worldwide subscribers to subscribers in the sample. Due to the nature of our sample, this ratio will be the same for all services.

\section{Conclusions}

At the ECOC symposium, estimates will be presented on the global power consumption of communication networks. In analogy with a previous study ${ }^{4}$, these results will be combined with other power consumption values, to get an idea of the electricity consumption of ICT in general. The following categories will be discussed:

- communication networks

- personal computers (desktops, laptops, computer screens)

- data centers

- mobile devices

\section{Acknowledgements}

The research leading to these results has received funding from the European Community's Seventh Framework Programme (FP7/2007-2013) under grant agreements $n^{\circ}$ 288021 (Network of Excellence "EINS") and $\mathrm{n}^{\circ}$ 257740 (Network of Excellence "TREND"). Moreover, this work has been carried out with the financial support of the Interdisciplinary institute for Broadband Technology (IBBT) through the IBBT-ISBO project "Energy efficiency in and by ICT".

\section{References}

[1] M. A. Marsan et al., IEEE International Conference on Communications Workshops, 1-5 (2009).

[2] W. Vereecken et al., IEEE Communications Magazine 49, 62 (2011).

[3] K. Hinton et al., IEEE Network 25, 6 (2011).

[4] M. Pickavet et al., Proc. ANTS'08 (2008).

[5] M. Webb et al., Smart 2020: Enabling the low carbon economy in the information age, The Climate Group (2008).

[6] D. Kilper et al., IEEE Journal of Selected Topics in Quantum Electronics 17, 275 (2011)

[7] J. Baliga et al., IEEE Communications Magazine 49, 70 (2011)

[8] ITU World Telecommunication / ICT Indicators database, ITU (2012).

[9] World Population Prospects: The 2010 Revision, UN Population Division (2011).

[10] http://blog.telegeography.com/post/285520 02503, GlobalComms Pay-TV Research Service (2012).

[11] G. Hardy et al., Better Viewing, Lower Energy Bills, and Less Pollution: Improving the Efficiency of Television Set-Top Boxes, NRDC (2011).

[12] Vodafone annual report 2011. 